\title{
Distributed Source Localization Based on TOA Measurements in Wireless Sensor Networks
}

\author{
Wanzhi Qiu and Efstratios Skafidas \\ National ICT Australia, Department of Electrical and Electronic Engineering, The University of Melbourne, \\ Parkville, Victoria 3010, Australia \\ Correspondence should be addressed to Wanzhi Qiu, wanzhiqiu128@yahoo.com
}

Received 30 October 2008; Accepted 30 December 2008

Recommended by Muhammad Taher Abuelma'atti

\begin{abstract}
We study the problem of source localization in multihop wireless sensor networks. A fully distributed algorithm based on sensor measurements of time of arrivals (TOAs) is proposed. In contrast to centralized methods where all TOA measurements are transmitted via certain routes to a central location (the sink) for processing, the proposed method distributes the processing among the relay nodes on the routes to the sink. Fusion strategies are proposed so that the raw and intermediate data are progressively processed, and only the refined results are further relayed. As a result, the proposed scheme has improved flexibility and scalability since it does not impose any special requirements on the sink node. The proposed distributed strategy also has the potential to save energy and bandwidth due to reduced radio transmissions.
\end{abstract}

Copyright ( $) 2009$ W. Qiu and E. Skafidas. This is an open access article distributed under the Creative Commons Attribution License, which permits unrestricted use, distribution, and reproduction in any medium, provided the original work is properly cited.

\section{Introduction}

In centralized wireless sensor network (WSN) source localization, the transmission of all the raw measurements to a central point is required and the processing is performed in a centralized manner. These schemes have the potential to achieve optimum solutions due to the availability of the full data set at the processing point. However, the centralized systems typically have low energy efficiency and low scalability due to excessive radio transmissions and dependency on a central processing node.

Distributed methods based on clustering have been proposed [1]. In [2], the intermediate result is cycled through the network on specially designed routes, and each node makes a small adjustment to the received estimate based on deepest descent on a cost function, then passes updated estimate to one of its neighbours.

In this paper, we propose a fully distributed source localization algorithm based on TOA measurements in multihop wireless sensor networks. The proposed scheme distributes the localization process among the relay nodes along the routes to the (arbitury) node where the localization estimate is needed. Whenever determined feasible, the intermediate estimate is produced or refined by incorporating TOAs and intermediate estimates available at the local node. Data fusion strategies are proposed so that the raw and intermediate data are progressively processed and only the refined results are further relayed.

\section{The Data Model and Centralized Processing}

As in [3] and illustrated in Figure 1, for a signal emitted by the source at time $T$ (unknown), the TOA measurement at node $i(i=1, \ldots, N, N \geq 3)$ is modelled by

$$
t_{i}=T+\frac{R_{i}}{c}+\varepsilon_{i}, \quad i=1, \ldots, N
$$

where $\varepsilon_{i}$ denotes measurement noise and $c$ the propagation speed. The noise samples are assumed to be uncorrelated with zero mean and variance $\sigma^{2}$. No assumption is made about the probability of density function of the noise. The distance between the source and a node located at $\left(x_{i}, y_{i}\right)$ is given by $R_{i}=\sqrt{\left(x-x_{i}\right)^{2}+\left(y-y_{i}\right)^{2}}$.

We assume that a nominal source position $\left(x_{n}, y_{n}\right)$ is available. This nominal position, which is close to the true 


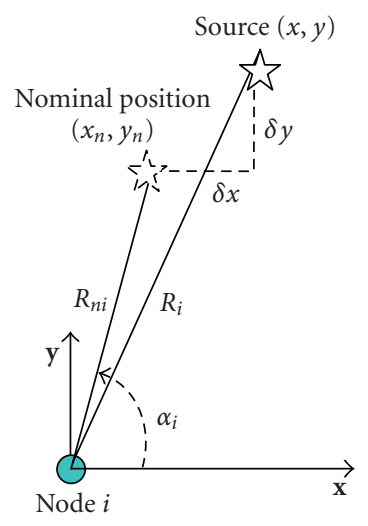

FIGURE 1: Source localization geometry.

source position $(x, y)$, could have been obtained from the previous measurements. Such a situation is typical when the source is being tracked [3]. Hence, to estimate the source position we require an estimation of $\left[\left(x-x_{n}\right)\left(y-y_{n}\right)\right]^{T}=$ $\left[\begin{array}{ll}\delta x & \delta y\end{array}\right]^{T}$, where $T$ denotes matrix transpose.

Using a first-order Taylor expansion of $R_{i}$ about $\left(x_{n}, y_{n}\right)$ and defining $R_{n i}=\sqrt{\left(x_{n}-x_{i}\right)^{2}+\left(y_{n}-y_{i}\right)^{2}}$, we have $R_{i} \approx$ $R_{n i}+\left(x_{n}-x_{i}\right) R_{n i} \delta x+\left(y_{n}-y_{i}\right) R_{n i} \delta y$. Therefore, (1) can be expressed as

$$
\tau_{i} \approx T+\frac{\cos \alpha_{i}}{c} \delta x+\frac{\sin \alpha_{i}}{c} \delta y+\varepsilon_{i}
$$

where $\cos \alpha_{i}=\left(x_{n}-x_{i}\right) R_{n i}, \quad \sin \alpha_{i}=\left(y_{n}-y_{i}\right) R_{n i}$, and $\tau_{i}=$ $t_{i}-R_{n i} / c$.

By collecting all TOA measurements at one point, the centralized processing scheme formulates the model $x=\mathbf{H} \boldsymbol{\theta}+\mathbf{w}$, where $\mathbf{x}=\left[\begin{array}{llll}\tau_{1} & \tau_{2} & \cdots & \tau_{N}\end{array}\right]^{T}, \boldsymbol{\theta}=$ $\left[\begin{array}{lll}T & \delta x & \delta y\end{array}\right]^{T}, \mathbf{w}=\left[\begin{array}{llll}\varepsilon_{1} & \varepsilon_{2} & \cdots & \varepsilon_{N}\end{array}\right]^{T}$, and

$$
\mathbf{H}=\left[\begin{array}{ccc}
1 & \frac{\cos \alpha_{1}}{c} & \frac{\sin \alpha_{1}}{c} \\
1 & \frac{\cos \alpha_{2}}{c} & \frac{\sin \alpha_{2}}{c} \\
\vdots & \vdots & \vdots \\
1 & \frac{\cos \alpha_{N}}{c} & \frac{\sin \alpha_{N}}{c}
\end{array}\right] .
$$

Based on this linear model, the unknowns $T, \delta x$, and $\delta y$ can be solved by the best linear unbiased estimator (BLUE) $\boldsymbol{\theta}=$ $\left[\mathbf{H}^{T} \mathbf{H}\right]^{-1} \mathbf{H}^{T} \mathbf{X}$, and the estimation variances are given by the diagonal items of the covariance matrix $\mathbf{C}_{\boldsymbol{\theta}}=\sigma^{2}\left[\mathbf{H}^{T} \mathbf{H}\right]^{-1}$, that is, $\left[\sigma_{T}^{2}, \sigma_{\delta x}^{2}, \sigma_{\delta y}^{2}\right]=\left[\mathbf{C}_{\theta}(1,1), \mathbf{C}_{\theta}(2,2), \mathbf{C}_{\theta}(3,3)\right]$.

Note that, in order to perform the estimation, the sink, where the processing is performed, has to know the locations of all participating nodes as well as their TOA measurements. This could cause severe communication/energy problems in situations when, for example, nodes are moving and therefore being required to constantly report their locations to the sink.

\section{The Proposed Distributed Processing}

As with the centralized processing, we assume that the sink is the point where the final estimate is needed. However, in the proposed scheme, the sink is just an ordinary node which does not have additional energy or computing capacity and does not perform any additional work as compared to other nodes. Only the nodes which have a TOA measurement or have received any measurements or intermediate estimates (IEs) from the up-stream nodes participate in the process. The principle is, for each participating node, to process the available measurements and/or IEs whenever possible and to send the result to the next-hop node (i.e., the down-stream node). The fusion result from the sink node is the final estimate. Note that the proposed scheme does not require any special arrangements on other functionalities of the network such as routing; TOA measurements and IEs are fused naturally while travelling on any established routes to the sink.

Different fusion rules have to be used depending on the information available at a node. Basically, three or more TOA measurements can be fused into one IE where a TOA measurement is defined as the location of the node and its measured $\tau_{i}$, that is, TOA $\triangleq\left(x_{i}, y_{i}, \tau_{i}\right)$. The IE is defined as the estimates of the unknowns and their corresponding variances, that is, IE $=\left(\widehat{\delta x}, \widehat{\delta y}, \widehat{\delta_{T}}, \sigma_{\delta x}^{2}, \sigma_{\delta y}^{2}, \sigma_{T}^{2}\right)$. An IE can be further fused with one measurement, two measurements, or one or more IEs. Fusion is performed at a local node after receiving all TOAs and/or IEs from up-stream nodes. When an IE is obtained, all follow-on transmission on this particular route will involve one IE only. Figure 2 depicts a typical fusion scenario where the arrows represent data transmissions, and rectangular blocks indicate performed fusion functions which are described in detail below.

3.1. Fuse_TOAs(). This function fuses $N(\geq 3)$ TOA measurements into one IE. The formulas used here are exactly the same as in Section 2.

3.2. Fuse_IEs(). When $N(>1)$ IEs are available, they can be combined according to the principle of BLUE to yield an updated IE. That is, for $\left.\widehat{(\delta x n}, \widehat{\delta y n}, \widehat{\delta_{T n}}, \sigma_{\delta x n}^{2}, \sigma_{\delta y n}^{2}, \sigma_{T n}^{2}\right)$, where $n=1, \ldots, N$, the updated IE is given by $\delta x_{-}$new $=$ $\sigma_{\delta x_{-} \text {new }}^{2} \sum_{n=1}^{N} \widehat{\delta x n} / \sigma_{\delta x n}^{2}, \delta y_{-}$new $=\sigma_{\delta y_{-} \text {new }}^{2} \sum_{n=1}^{N} \widehat{\delta y n} / \sigma_{\delta y n}^{2}$, and $\widehat{T n} \_$new $=\sigma_{T \_ \text {new }}^{2} \sum_{n=1}^{N} \widehat{T n} / \sigma_{T n}^{2}$, where $\sigma_{\delta x \text { nnew }}^{2}=$ $\left(\sum_{n=1}^{N} 1 / \sigma_{\delta x n}^{2}\right)^{-1}, \sigma_{\delta y_{\text {nnew }}}^{2}=\left(\sum_{n=1}^{N} 1 / \sigma_{\delta y n}^{2}\right)^{-1}$, and $\sigma_{T \text { _new }}^{2}=$ $\left(\sum_{n=1}^{N} 1 / \sigma_{T n}^{2}\right)^{-1}$.

As can be seen from the above equations, the BLUE is actually a weighted average of the estimates, giving the more accurate (with smaller variances) ones larger weights.

3.3. Fuse_IE_with_one_TOA(). We now fuse one intermediate estimate IE $0=\left(\widehat{\delta x}, \widehat{\delta y}, \widehat{T}, \sigma_{\delta x}^{2}, \sigma_{\delta y}^{2}, \sigma_{T}^{2}\right)$ with one TOA $\left(x_{i}, y_{i}, \tau_{i}\right)$ measured by a certain node $n_{i}$. In other words, we refine IE 0 with the new information provided by the TOA measurement. In order to do this, we approximate $T$ by its 


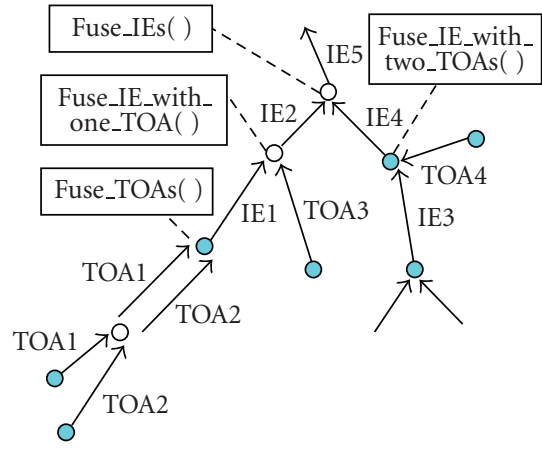

O Nodes with own TOA measurements

O Nodes without own TOA measurements

Figure 2: A typical fusion scenario.

estimated value $\hat{T}$ and refine the estimates of $\delta x$ and $\delta y$ only. By denoting $T \approx \widehat{T}+\varepsilon_{T}$, where $\varepsilon_{T}$ is the error in the estimate of $T$, the TOA measurement (2) can be expressed by $\tau_{i}-\hat{T} \approx\left(\cos \alpha_{i} / c\right) \delta x+\left(\sin \alpha_{i} / c\right) \delta y+\varepsilon_{i}+\varepsilon_{T}$ which can be further simplified as

$$
z=a \delta x+b \delta y
$$

where $a=\cos \alpha_{i} / c, b=\sin \alpha_{i} / c$, and

$$
z=z_{o}+\varepsilon_{i}+\varepsilon_{T}, \quad z_{o}=\tau_{i}-\widehat{T} .
$$

When the error terms $\left(\varepsilon_{i}\right.$ and $\left.\varepsilon_{T}\right)$ are ignored, (4) can be represented by a straight line in the $\delta x-\delta y$ plane. The estimate $(\widehat{\delta x}, \widehat{\delta y})$ is a point on the same plane, and we propose to use a weighted average of it and its projection onto line (4) $\left(\widehat{\delta x^{\prime}}, \widehat{\delta y^{\prime}}\right)$ as the new estimate. As far as the node $n_{i}$ can see, $\left(\widehat{\delta x^{\prime}}, \widehat{\delta y^{\prime}}\right)$ lies on the circle centred at $\left(x_{i}, y_{i}\right)$ with radius $r=c z_{0}$, where $c$ is the propagation speed. It can be seen from (5) that the variance of $r$ is given by $\sigma_{r}^{2}=c^{2}\left(\sigma^{2}+\right.$ $\left.\sigma_{T}^{2}\right)$. Since it is too complex to obtain the exact expressions for the variances of $\widehat{\delta x^{\prime}}$ and $\widehat{\delta y^{\prime}}$, in order to determine their weights in forming the new estimates, we will use $\sigma_{r}^{2}$ to approximate their variances, that is, $\sigma_{\delta x^{\prime}}^{2} \approx \sigma_{\delta y^{\prime}}^{2} \approx \sigma_{r}^{2}$. This approximation is in line with the new information the TOA measurement provides on the source location.

By denoting $K=a^{2}+b^{2}$, the projection coordinates can be expressed by

$\left(\widehat{\delta x^{\prime}}, \widehat{\delta y^{\prime}}\right)=\left(\frac{a}{K} z_{o}+\frac{b^{2}}{K} \widehat{\delta x}-\frac{a b}{K} \widehat{\delta y}, \frac{b}{K} z_{o}-\frac{a b}{K} \widehat{\delta x}+\frac{a^{2}}{K} \widehat{\delta y}\right)$,

leading to new estimates

$$
\begin{aligned}
& \delta x \_ \text {new }=\sigma_{\delta x \_ \text {new }}^{2}\left(\frac{\widehat{\delta x}}{\sigma_{\delta x}^{2}}+\frac{\widehat{\delta x^{\prime}}}{\sigma_{\delta x^{\prime}}^{2}}\right), \\
& \delta y_{\text {nnew }}=\sigma_{\delta y_{\text {nnew }}}^{2}\left(\frac{\widehat{\delta y}}{\sigma_{\delta y}^{2}}+\frac{\widehat{\delta y^{\prime}}}{\sigma_{\delta y^{\prime}}^{2}}\right),
\end{aligned}
$$

with updated variances $\sigma_{\delta x_{-} \text {new }}^{2}=\left(1 / \sigma_{\delta x}^{2}+1 / \sigma_{\delta x^{\prime}}^{2}\right)^{-1}$ and $\sigma_{\delta y_{\text {new }}}^{2}=\left(1 / \sigma_{\delta y}^{2}+1 / \sigma_{\delta y^{\prime}}^{2}\right)^{-1}$.

3.4. Fuse_IE_with_two_TOAs(). Here, we have two TOA measurements $\left(x_{1}, y_{1}, \tau_{1}\right)$ and $\left(x_{2}, y_{2}, \tau_{2}\right)$ along with one IE IE $0=\left(\widehat{\delta x}, \widehat{\delta y}, \widehat{T}, \sigma_{\delta x}^{2}, \sigma_{\delta y}^{2}, \sigma_{T}^{2}\right)$ available. We first use the BLUE principle to fuse the two TOAs to obtain initial estimates $\widehat{\delta x^{\prime}}$ and $\widehat{\delta y^{\prime}}$. The result is then further fused with IE 0 . As in the previous section, by denoting $T \approx \hat{T}+\varepsilon_{T}$, for $i=1,2$, we have $z_{i}=a_{i} \delta x+b_{i} \delta y+\varepsilon_{i}+\varepsilon_{T}$, where $a_{i}=\cos \alpha_{i} / c$, $b_{i}=\sin \alpha_{i} / c$, and $z_{i}=\tau_{i}-\widehat{T}$.

By defining $\mathbf{x}=\left[\begin{array}{ll}z_{1} & z_{2}\end{array}\right]^{T}, \mathbf{H}=\left[\begin{array}{cc}a_{1} & b_{1} \\ a_{2} & b_{2}\end{array}\right], \boldsymbol{\theta}=\left[\begin{array}{ll}\delta x & \delta y\end{array}\right]^{T}$, and $\mathbf{w}=\left[\begin{array}{ll}\varepsilon_{1}+\varepsilon_{T} & \varepsilon_{2}+\varepsilon_{T}\end{array}\right]^{T}$, we have $x=\mathbf{H} \boldsymbol{\theta}+\mathbf{w}$ which immediately leads to the following BLUE [3]:

$$
\left[\widehat{\delta x^{\prime}}, \widehat{\delta y^{\prime}}\right]^{T}=\left[\mathbf{H}^{T} \mathbf{C}^{-1} \mathbf{H}\right]^{-1} \mathbf{H}^{T} \mathbf{C}^{-1} \mathbf{x},
$$

with covariance matrix $\mathbf{C}_{\boldsymbol{\theta}}=\left[\mathbf{H}^{T} \mathbf{C}^{-1} \mathbf{H}\right]^{-1}$, where, with $E$ denoting expectation,

$$
\mathbf{C}=E\left(\mathbf{w} \mathbf{w}^{T}\right)=\left(\begin{array}{cc}
\sigma^{2}+\sigma_{T}^{2} & \sigma_{T}^{2} \\
\sigma_{T}^{2} & \sigma^{2}+\sigma_{T}^{2}
\end{array}\right) .
$$

That is, $\left[\sigma_{\delta x^{\prime}}^{2}, \sigma_{\delta y^{\prime}}^{2}\right]=\left[\mathbf{C}_{\theta}(1,1), \mathbf{C}_{\theta}(2,2)\right]$. This fusion function is now summarized as follows. Step 1, use (8) to obtain $\widehat{\delta x^{\prime}}$ and $\widehat{\delta y^{\prime}}$. Step 2, fuse $\left(\widehat{\delta x^{\prime}}, \widehat{\delta y^{\prime}}\right)$ and $(\widehat{\delta x}, \widehat{\delta y})$ using (7).

\section{Simulation}

In this section, we conduct simulation to verify the proposed distributed source localization scheme. The tree routing protocol and network setup process including parent-child relationship and address assignment algorithms of the ZigBee [4] standard are followed in the simulation.

100 ZigBee nodes are randomly deployed in a square region with size $500 \mathrm{~m}$ by $500 \mathrm{~m}$. The root node (the coordinator in ZigBee's terms) is acting as the sink and is located at the centre of the lower edge of the region. The nominal location of the sound source (target) (with speed $c=344 \mathrm{~m} / \mathrm{s}$ ) is the centre of the region, and it has an effective range $R=200 \mathrm{~m}$. When a node is less than $R$ meters away from the target, it can make a TOA measurement. For each simulation scenario, the source takes a small move in both $x$ and $y$ directions, that is, $(\delta x, \delta y)=$ $(0.5 \mathrm{~m}, 0.5 \mathrm{~m})$, and 1000 runs are conducted with independent and identically distributed zero-mean Gaussian noise samples generated in each run. The estimation accuracy is measured by the mean square error over all the runs: MSE $=$ $(1 / 1000) \sum_{r=1}^{1000}\left(\left(x-\hat{x}_{r}\right)^{2}+\left(y-\hat{y}_{r}\right)^{2}\right)$, where $\left(x_{r}, y_{r}\right)$ is the estimate of the true source location $(x, y)$ in the $r$ th run.

Figure 3 shows the estimation errors with varying noise variance. It can be seen that the proposed distributed scheme works, and its performance attains that of the centralized best linear unbiased estimator when the noise is reduced to a certain level. 


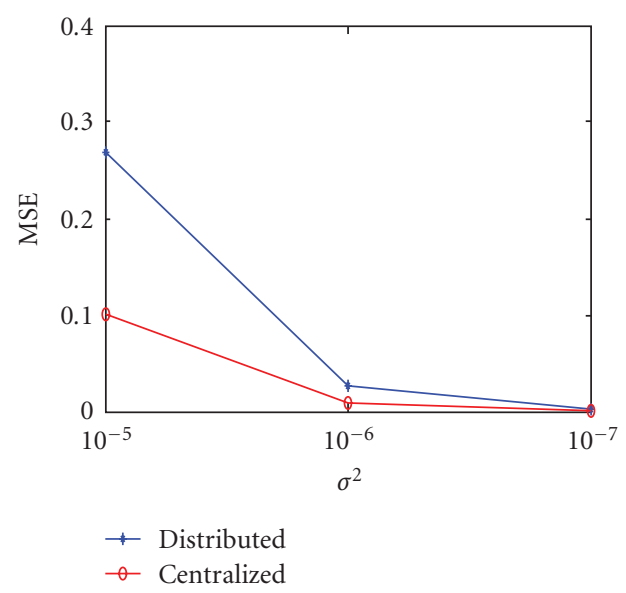

FIGURE 3: MSE of estimates.

In summary, the benefit of the proposed scheme is twofold. First, it has improved flexibility and scalability over centralized schemes since it shares the processing among multiple nodes and does not pose any special requirements on the sink node. Second, it has the potential to save energy/bandwidth consumption of the network due to reduced radio transmissions.

\section{References}

[1] W.-P. Chen, J. C. Hou, and L. Sha, "Dynamic clustering for acoustic target tracking in wireless sensor networks," in Proceedings of the 11th IEEE International Conference on Network Protocols (ICNP '03), pp. 284-294, Atlanta, Ga, USA, November 2003.

[2] M. G. Rabbat and R. D. Nowak, "Decentralized source localization and tracking," in Proceedings of the IEEE International Conference on Acoustics, Speech and Signal Processing (ICASSP '04), vol. 3, pp. 921-924, Montreal, Canada, May 2004.

[3] S. M. Kay, Fundamentals of Statistical Signal Processing: Estimation Theory, Prentice-Hall, Englewood Cliffs, NJ, USA, 1993.

[4] ZigBee Specification Version 1.0, ZigBee Alliance, 2005. 

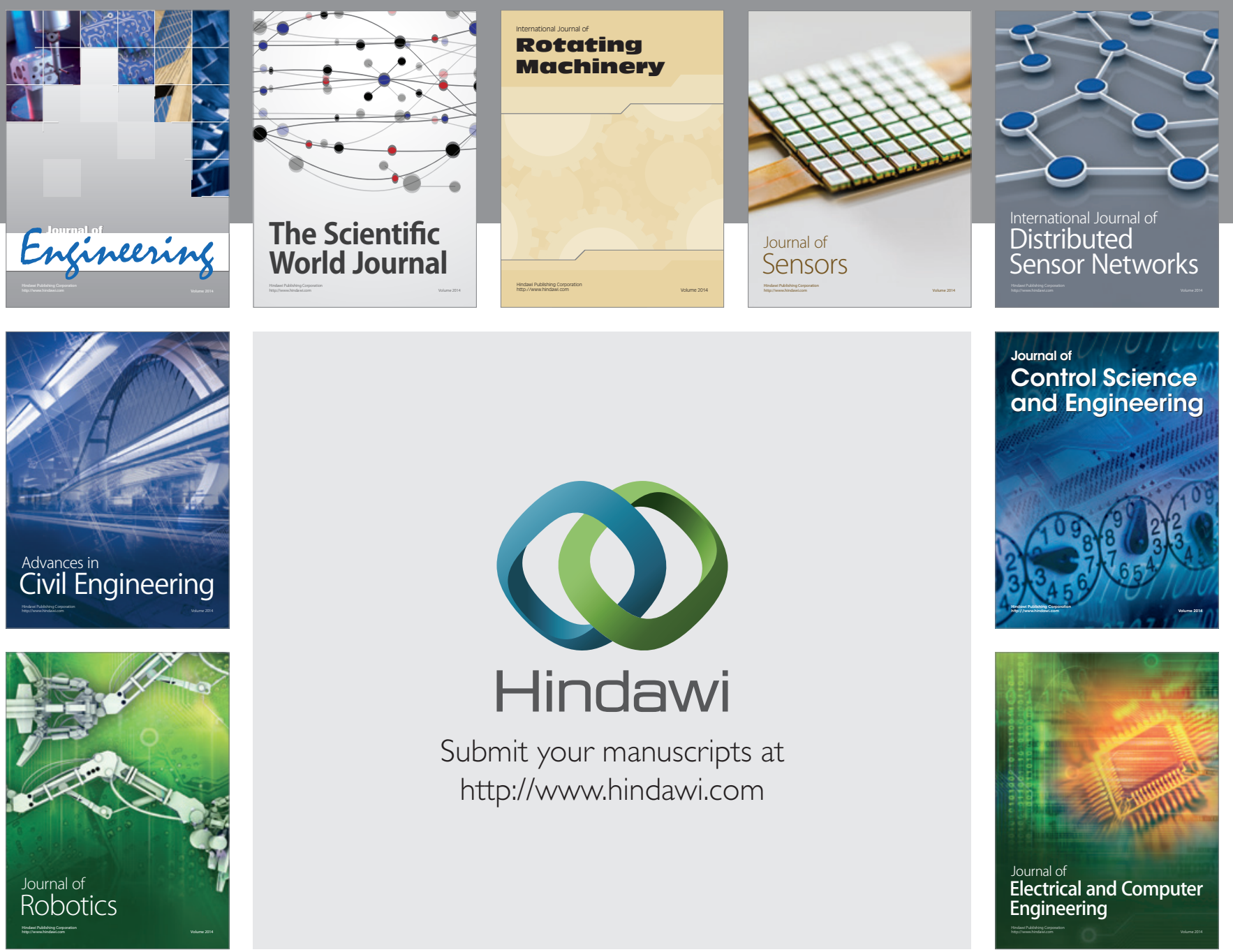

Submit your manuscripts at

http://www.hindawi.com
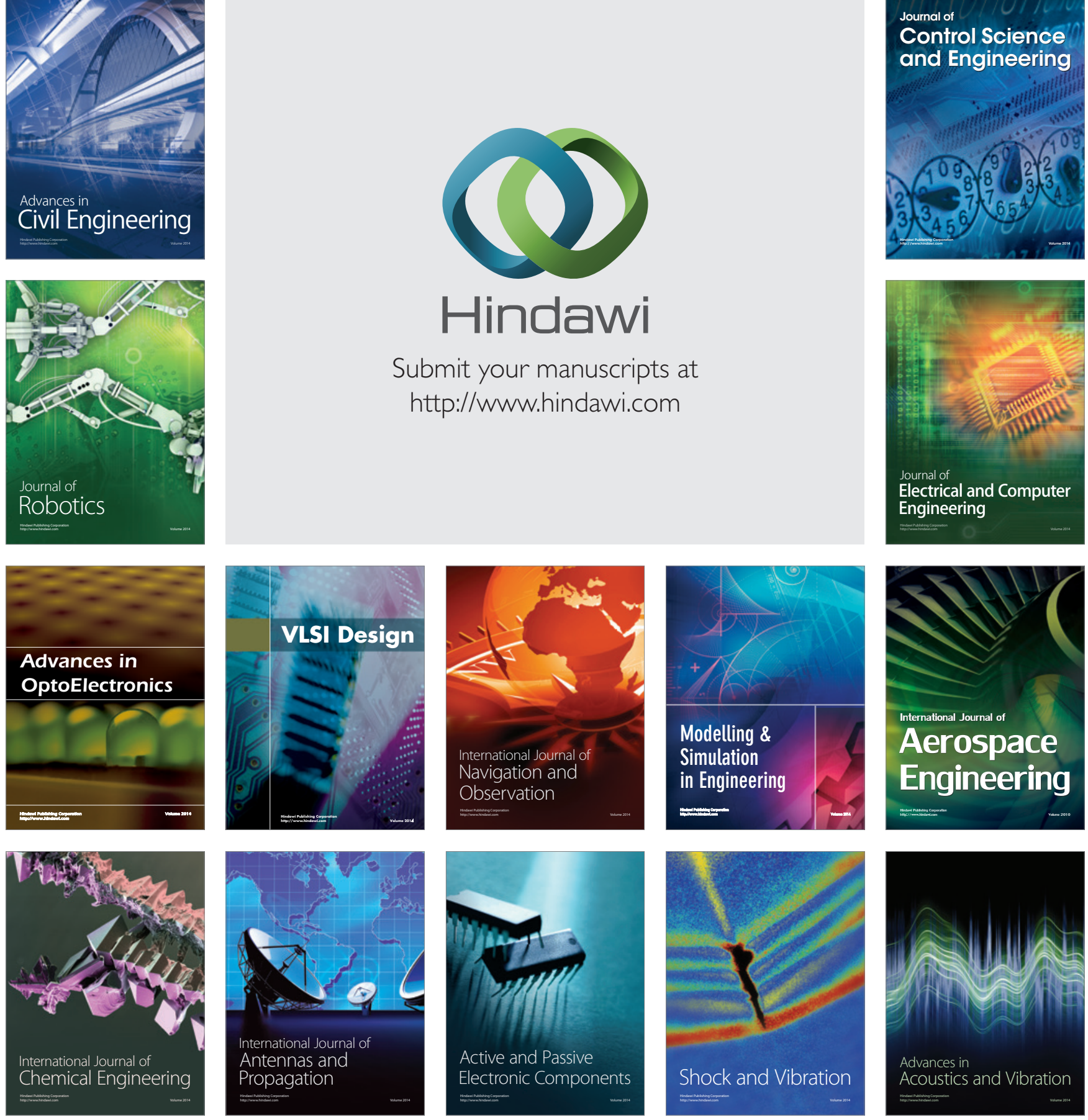